\title{
Laminine 5, migration cellulaire et cancer
}

Patricia Rousselle

$>$ La laminine 5 est exprimée dans les lames basales des épithéliums squameux et transitionnels spécialisés. Ses propriétés structurales lui permettent de maintenir la cohésion épithéliomésenchymateuse. Elle induit des signaux biologiques déterminants, responsable de l'assemblage des structures d'adhérence cellulaires stables que sont les hémidesmosomes. Des études récentes montrent son implication dans la migration des cellules métastatiques au cours de la progression tumorale et cet article fait le point sur les différentes hypothèses proposées à ce jour. <

Les interactions entre les cellules et la matrice extracellulaire (MEC) jouent un rôle important dans le contrôle du comportement cellulaire. Ce contrôle s'effectue par des interactions spécifiques entre les molécules matricielles et les cellules grâce à des récepteurs transmembranaires, principalement de la famille des intégrines, dont le domaine intracellulaire est relié aux constituants du cytosquelette. Cela permet à la matrice d'assurer la transmission dans la cellule de signaux qui vont moduler, selon les cas, l'adhérence, la migration, la prolifération, la différenciation ou l'apoptose. Ce contrôle du comportement cellulaire est crucial au cours du développement, mais, également lors des remaniements tissulaires physiologiques et pathologiques.

On distingue plusieurs types de matrice extracellulaire, selon les molécules qui les constituent et l'organisation qui en découle, les lames basales étant sans doute les plus spécialisées. Ces dernières sont de fins treillis protéiques continus sur lesquels reposent les différents feuillets cellulaires de l'organisme. Elles ont longtemps été définies comme des structures morphologiques à part dont la fonction était limitée au cloisonnement des compartiments tissulaires. C'est seulement au cours des quinze dernières années que,

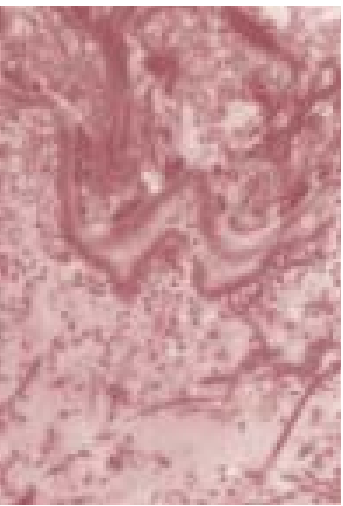

malgré leur faible représentativité et leur grande insolubilité, la composition moléculaire et les fonctions des lames basales ont été analysées en détail. Il apparaît que ces structures jouent un rôle fondamental dans le contrôle du comportement cellulaire, tant au cours du développement embryonnaire que dans le maintien de l'intégrité des phénotypes cellulaires différenciés (Figure 1).

Les laminines ( $L N)$, glycoprotéines principalement localisées dans les lames basales, représentent une famille de molécules dont les propriétés biologiques et structurales ont suscité un intérêt grandissant ces dernières années [1]. Identifiées au départ comme des constituants structuraux, il est clair aujourd'hui que les laminines entretiennent avec les cellules des relations étroites et leur transmettent des informations morphogénétiques importantes. Les laminines sont composées de trois chaînes polypeptidiques génétiquement distinctes, $\alpha, \beta$ et $\gamma$, reliées par des ponts disulfures. La structure primaire de 5 chaînes $\alpha$ ( $\alpha 1$ à $\alpha 5$ ), de 3 chaînes $\beta$ ( $\beta$ l à $\beta 3$ ) et de 3 chaînes $\gamma$ ( $\gamma \mathrm{l}$ à $\gamma 3$ ) a été déduite du séquençage des ADN complémentaires correspondants et les analyses biochimiques ont permis de mettre en évidence l'existence de 15 trimères différents (LN-1 à LN-15) [1]. L'expression et l'assemblage des différentes sous-unités 
varient de façon spatio-temporelle laissant présumer des rôles biologiques et structuraux spécifiques de chaque isoforme. Ces observations illustrent l'importance des laminines dans l'organisation tissulaire. Néanmoins, les mécanismes cellulaires et moléculaires $(\rightarrow) \mathrm{m} / \mathrm{s}$ $2000, n^{\circ} 12$ p. 1371 qui sont à la base de leurs fonctions spécifiques et de leurs rôles biologiques et structuraux ne sont pas complètement connus.

\section{L'ancrage des cellules épithéliales : un gage de stabilité assuré par la laminine 5}

La laminine 5 ( $L N-5)$ est spécifiquement exprimée dans les lames basales des épithéliums squameux et transitionnels spécialisés qui ont des fonctions de sécrétion ou de protection, comme les muqueuses ou la peau. La $\mathrm{LN}-5$ résulte de l'assemblage hétérotrimérique des sous-unités $\alpha 3, \beta 3$ et $\gamma 2$ et est synthétisée exclusivement par les cellules épithéliales sous la forme d'un précurseur de 460 kDa (Figure 2). Les parties carboxyterminales des trois chaînes sont assemblées sous la

$(\rightarrow) \mathrm{m} / \mathrm{s}$ 2002, $n^{\circ} 5$, p. 519 forme d'une hélice $\alpha$ super-enroulée et la chaîne $\alpha 3$, plus longue que les deux autres, comporte un domaine globulaire constitué de 5 répétitions de résidus basiques (globules LGl à LG5). Dans les conditions physiologiques, chacune des sous-unités $\alpha 3$ et $\gamma 2$ subit un clivage posttraductionnel extracellulaire de ses extrémités carboxy- et amino-terminale respectivement, aboutissant à la forme tissulaire mature [2]. Un clivage supplémentaire d'un fragment de $20 \mathrm{kDa}$ se produit également, dans certaines conditions, à l'extrémité amino-terminale de la chaîne $\alpha 3[2,3]$. La plasmine et la BMPl (bone morphogenetic protein-1) ont été initialement tenues pour responsables de la coupure du module LG4-LG5 (LG4/5) de la chaîne $\alpha 3[3,4]$. Toutefois, la caractérisation récente du site de clivage contredit cette hypothèse et indique qu'une enzyme de la famille des métalloprotéases matricielles (MMP) en serait l'auteur $(\rightarrow)$ [5]. La BMPl est l'enzyme responsable du clivage de la chaîne $\gamma 2$ [3], et l'intervention simultanée d'isomérases spécifiques est nécessaire au remaniement de certains ponts disulfures et au détachement définitif du fragment amino-terminal [6]. Des études récentes indiquent que la totalité de la chaîne $\gamma 2$ sous sa forme de précurseur est nécessaire à la sécrétion et à l'intégration de la LN-5 dans la matrice extracellulaire [7]. Le rôle majeur de la LN-5 est souligné par l'existence de maladies héréditaires ou acquises, résultant d'une anomalie de synthèse et/ou d'expression de l'une de ses sous-unités constitutives. Ces maladies, appelées épidermolyses bulleuses jonctionnelles, conduisent notamment à une fragilité de la jonction dermo-épidermique de la peau caractérisée par la formation spontanée de bulles épidermiques $(\rightarrow)$. La LN-5 joue ainsi un rôle crucial et irremplaçable dans la cohésion épithélio-mésenchymateuse. Elle est associée de façon covalente à la LN-6 ( $\alpha 3 \beta 1 \gamma 1)$ [2], formant ainsi une véritable charpente moléculaire, cible de multiples interactions. Par ailleurs, I'ancrage de la LN-5 au stroma sous-jacent est assuré par la liaison de ses bras courts aux extrémités globulaires du collagène VII, composant majeur des fibrilles d'ancrage [8].

La LN-5 a un rôle biologique déterminant puisqu'elle permet l'adhérence des cellules épithéliales adjacentes par l'intermédiaire des intégrines et induit l'assemblage des structures d'adhérence stable que sont les hémidesmosomes. Le site d'adhérence et de liaison aux intégrines $\alpha 3 \beta 1$ et $\alpha 6 \beta 4$ est localisé sur les globules LG1-LG2-LG3 $[9,10]$. De nombreux travaux montrent que c'est l'interaction de l'intégrine $\alpha 6 \beta 4$ avec la LN-5 mature qui déclenche l'assemblage des hémi-

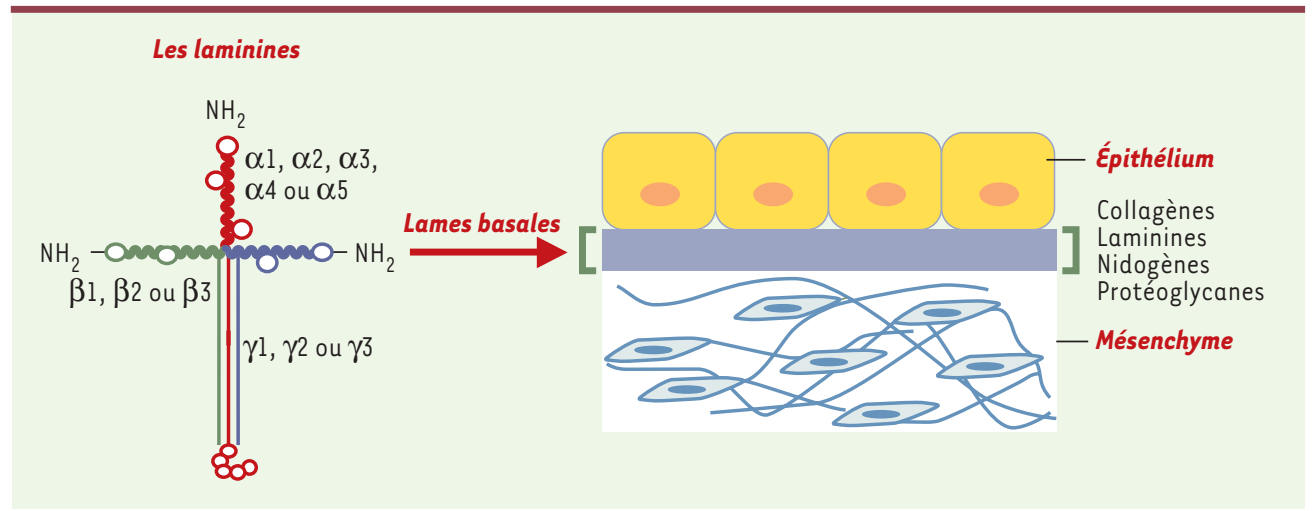

Figure 1. Illustration schématique de la structure des lames basales. Les membres de la famille des laminines sont des constituants majeurs des lames basales, fins feuillets de matrice extracellulaire au contact des cellules ou localisés à l'interface entre un épithélium et un tissu conjonctif. Les laminines comportent trois chaînes génétiquement distinctes, $\alpha, \beta$ et $\gamma$, reliées par des ponts disulfures. Cinq chaînes $\alpha(\alpha 1$ à $\alpha 5)$, trois chaînes $\beta$ ( $\beta 1$ à $\beta 3$ ) et trois chaînes $\gamma(\gamma l$ à $\gamma 3$ ) ont été identifiées chez les mammifères et le nombre connu de combinaisons est de 12 trimères différents (LN-1 à LN-12) ayant chacun une localisation tissulaire précise. À noter qu'outre les laminines, les lames basales comportent d'autres composants matriciels comme les collagènes IV, les nidogènes et des protéoglycanes. Ces molécules interagissent et forment des assemblages moléculaires précis conférant à ces tissus une extrême solidité. 
desmosomes intracellulaires [10]. La LN-5 mature constituerait ainsi une barrière à la motilité cellulaire $[4,11]$. Le rôle exact de l'intégrine $\alpha 3 \beta 1$ n'est pas connu. Sa participation au contrôle de la prolifération et/ou de la migration cellulaire a été proposée. Une fonction dans l'organisation des composants de la lame basale lui a été attribuée ainsi qu'une participation au processus d'activation de certaines enzymes $[9,12]$.

\section{La migration des cellules épithéliales : la dissolution de l'ancrage stable}

Un certain nombre de situations biologiques comme le développement embryonnaire, les réponses immunitaires, la réparation tissulaire et l'invasion tumorale impliquent des mouvements cellulaires et mettent en jeu les mécanismes de migration cellulaire. La mise en place du processus de migration des cellules épithéliales induit des bouleversements phénotypiques et nécessite des interactions spécifiques avec les composants matriciels. La dissolution des hémidesmosomes est l'une des conséquences de ces nouvelles interactions, qui doivent êtres compatibles avec la migration cellulaire.

Les chercheurs ont rapidement compris que la $\mathrm{LN}-5$ non seulement assurait une adhérence stable, mais pouvait aussi jouer un rôle au cours de la migration

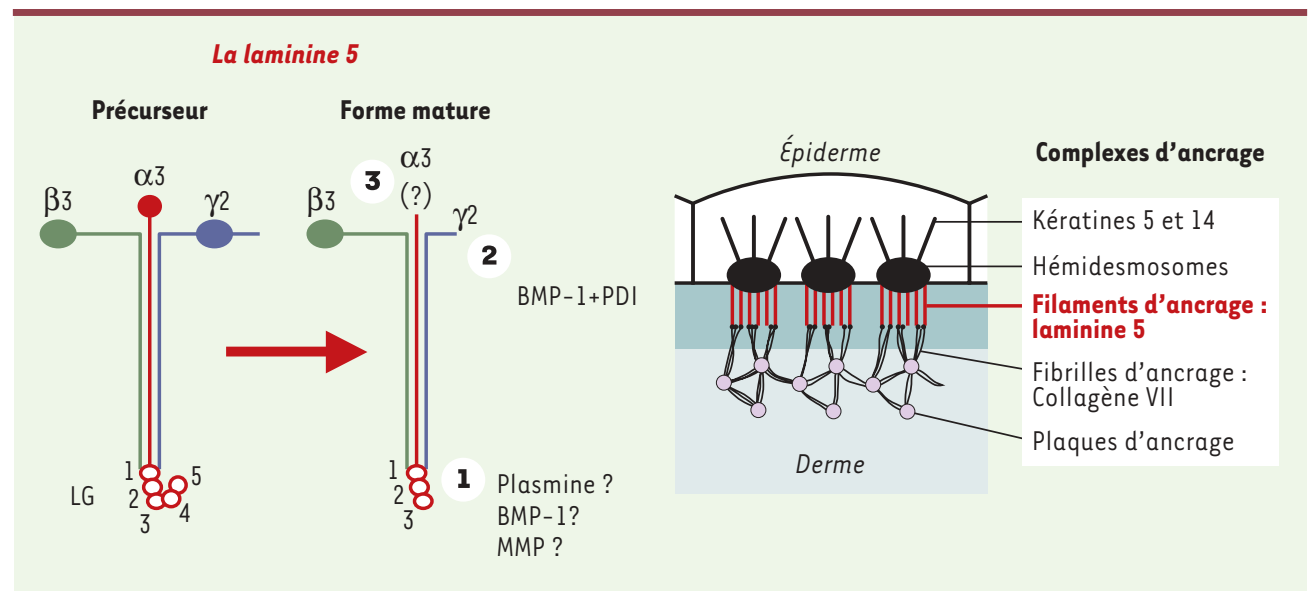

Figure 2. Maturation protéolytique et ancrage de la laminine 5. La LN-5 est synthétisée puis sécrétée par les cellules épithéliales sous la forme d'un précurseur. Dans les conditions physiologiques, les sous-unités $\alpha 3$ et $\gamma 2$ subissent un clivage post-traductionnel extracellulaire de leur extrémité carboxy-terminale pour $\alpha 3$ (1) et amino-terminale pour $\gamma 2$ (2). Ces clivages aboutissent à la forme tissulaire mature. Un clivage supplémentaire de l'extrémité amino-terminale de la sous-unité $\alpha 3$ a également été décrit (3). La LN-5 tissulaire a été décrite comme jouant un rôle crucial et irremplaçable dans la cohésion dermo-épidermique. De plus, elle transmet des signaux biologiques déterminants puisqu'elle permet l'adhérence des kératinocytes, par l'intermédiaire de récepteurs membranaires de la famille des intégrines, et induit l'assemblage des structures d'adhérence stable que sont les hémidesmosomes. BMP-1 : bone morphogenetic protein 1 ; MMP : métalloprotéases matricielles. des cellules épithéliales. Les premiers indices rapportaient une expression et une synthèse accrues de la LN-5 par des kératinocytes mobiles in vitro [13]. Des études in vivo de la cicatrisation cutanée ont montré une expression accrue des différentes chaînes de la LN-5 par les kératinocytes localisés dans la zone de colonisation de la plaie [14]. La LN-5 sous sa forme de précurseur a ainsi été identifiée comme le composant matriciel majeur au contact des kératinocytes migrants, indiquant que l'absence de clivage des chaînes $\alpha 3[4,15]$ et $\gamma 2$ [16] serait nécessaire à la migration de ces cellules. Ainsi, la molécule précurseur permettrait la migration des kératinocytes en empêchant la formation des hémidesmosomes (Figure 3). Des études récentes indiquent qu'un récepteur membranaire de la famille des syndécanes [17] se lie spécifiquement au module LG4/5. De plus, l'intégrine $\alpha 2 \beta 1$ interagirait avec la chaîne $\gamma 2$ sous forme de précurseur au cours de la migration cellulaire [16].

La complexité des mécanismes mis en œuvre au cours de la migration cellulaire est étayée par quelques résultats contradictoires. En effet, un ensemble de résultats a permis d'attribuer à la LN-5 mature la capacité d'induire la migration de certaines lignées cellulaires [18, 19]. L’habileté de ces cellules à migrer sur la $L N-5$ serait constitutive et liée à leur capacité d'exprimer spécifiquement les métalloprotéases matricielles, MMP2 ou MT1-MMP [19, 20]. Ces enzymes présentent la particularité de tronquer massivement la chaîne $\gamma 2$, ce qui démasquerait, selon les auteurs, un signal inducteur de la migration cellulaire. Ces observations rejoignent les résultats de travaux précédents montrant que la LN-5 ajoutée au milieu de culture entraîne la dispersion des cellules de ces mêmes lignées [21], effet qui n'est pas observé dans le cas des kératinocytes de la peau. Cet effet est donc strictement dépendant des cellules impliquées et de leur répertoire enzymatique. Si les mécanismes moléculaires à la base des interactions entre les cellules et la LN-5 au cours de la migration cellulaire sont encore 
imparfaitement compris, il apparaît toutefois que l'interaction de l'intégrine $\alpha 3 \beta$ l avec le module LG3 est déterminante aussi bien au cours de l'adhérence que de la migration cellulaire [22, 23].

\section{Laminine 5 et migration des cellules tumorales}

On désigne sous le nom de carcinomes des cancers issus de cellules épithéliales ayant perdu tout contrôle de leur prolifération et de leur capacité de se différencier. L'acquisition du caractère malin d'un carcinome se traduit par sa capacité de traverser la lame basale, d'envahir le tissu conjonctif sous-jacent et de fonder des métastases à distance de la tumeur initiale. Les interactions entre les cellules et la matrice extracellulaire jouent un rôle primordial dans les étapes initiales du processus invasif. Elles aboutissent à une augmentation de l'adhérence et de la migration cellulaire, mais également à la dégradation progressive de la matrice extracellulaire par un processus protéolytique. Des hypothèses ont été émises concernant la liaison spécifique possible des cellules sur les laminines lors de cette étape de progression tumorale.

Des travaux récents ont montré l'implication possible de la LN-5 dans le processus de malignité de certains carcinomes (adénocarcinomes du côlon, cancers gastriques, cancers de la prostate, du poumon, du pancréas et du col de l'utérus). Les études menées sur des tumeurs humaines ont montré une corrélation entre le caractère invasif de ces carcinomes et l'expression de la LN-5 par les cellules bourgeonnantes de ces tumeurs [24-26]. L'augmentation de synthèse de la LN- 5 par ces cellules constituerait, selon les auteurs, une étape clé nécessaire au déclenchement et au déroulement du processus migratoire invasif. La LN-5 néosynthétisée et sécrétée représenterait un substrat propice à la migration cellulaire.
Ainsi, l'expression accrue de la chaîne $\gamma 2$, examinée par des techniques d'immunohistochimie, reflèterait le fort potentiel invasif de ces cancers. Les anticorps monoclonaux spécifiques de cette protéine sont des outils actuellement utilisés pour établir le pronostic des patients atteints de ces cancers.

D'autres études rapportent au contraire, qu'une diminution de l'expression extracellulaire de la LN-5 serait à l'origine de la dissolution des hémidesmosomes des cellules épithéliales induisant une instabilité de l'adhérence et contribuant au plus grand potentiel invasif des cellules malignes [27]. Cette hypothèse découle de travaux menés sur des patients atteints de cancers de la prostate, du pancréas et du sein. Une étude rapporte qu'une anomalie de l'assemblage intracellulaire des sous-unités de la LN-5 serait à l'origine de l'accumulation de dimères $\beta 3 \gamma 2$ dans les cellules bourgeonnantes de certains cancers colorectaux [28, 29].

Toutes ces hypothèses résultent d'observations faites à partir de différentes tumeurs, et on ne connaît pour le moment ni le rôle précis de la LN-5, ni la forme

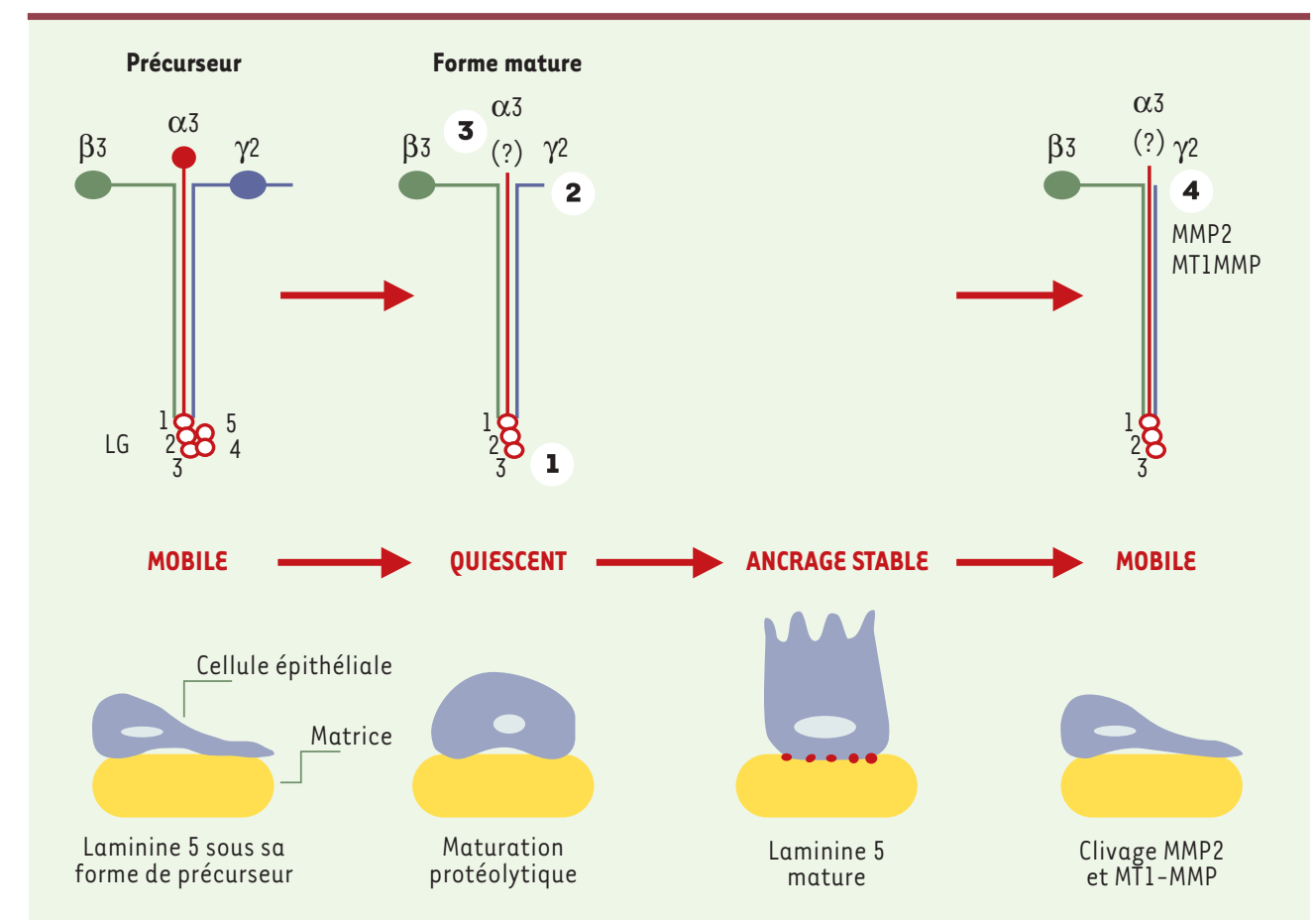

Figure 3. Deux formes moléculaires de la LN-5 sont associées à la migration cellulaire. La LN-5 sous sa forme de précurseur semble impliquée dans la migration de certaines cellules épithéliales : elle empêcherait la formation de structures d'ancrage stables par les intégrines. La maturation de la molécule permettrait de passer à un état stationnaire concrétisé par la formation des hémidesmosomes et un ancrage stable. Un clivage spécifique supplémentaire de la chaîne $\gamma 2$ par la métalloprotéase matricielle 2 (MMP2) a été mis en évidence dans certains systèmes cellulaires présentant une capacité accrue de migration. La signification biologique de ce clivage n'est pas connue. Cependant, il n'est pas exclu qu'il favorise la dissociation des assemblages propres à l'ancrage stable et qu'il permette, dans certaines circonstances, la mobilité cellulaire. 
moléculaire en cause dans ces mécanismes. II semble toutefois que, compte tenu de la complexité fonctionnelle de la $L N-5$, différents mécanismes d'action interviennent selon les cellules impliquées et leur environnement matriciel.

De même, les détails moléculaires du processus qui transforme une cellule tumorale en une cellule ayant acquis, de surcroît, des capacités d'invasion et de migration métastatique sont encore mal connus. Si la LN- 5 semble associée à cette étape clé du processus malin, elle n'est certainement pas seule responsable. Les récepteurs membranaires ainsi que les métalloprotéases exprimées par ces cellules jouent certainement un rôle crucial dans la perte de leur capacité d'adhérence au tissu d'origine et leur adressage vers d'autres cibles. Ces « acteurs extracellulaires » de la migration cellulaire constituent actuellement des cibles pour le développement de nouvelles stratégies thérapeutiques. $\diamond$

\section{SUMMARY}

Laminin 5, cell migration and cancer

Laminin 5 is an extracellular matrix protein found in basement membranes underlying specialised epithelia. It plays a crucial role by (1) inducing the assembly of hemidesmosomes through interactions with specific integrins and (2) supporting multiple connecting interactions with other components. Despite this role in stable adhesion, laminin 5 may play a role in cell migration and may act as a ligand for invading carcinoma cells. This review presents recent hypothesis supporting the involvement of laminin 5 in the tumor cascade and progression toward metastatic disease. $\diamond$

\section{RÉFÉRENCES}

1. Colognato H, Yurchenco PD. Form and function: the laminin family of heterotrimers. Dev Dyn $2000 ; 218: 213-34$.

2. Champliaud MF, Lunstrum GP, Rousselle P, Nishiyama T, Keene DR, Burgeson RE. Human amnion contains a novel laminin variant, laminin 7, which like laminin 6, covalently associates with laminin 5 to promote stable epithelial-stromal attachment. J Cell Biol 1996 ; 132 : 1189-98.

3. Amano S, Scott IC, Takahara K, et al. Bone morphogenetic protein $l$ is an extracellular processing enzyme of the laminin 5 gamma 2 chain. J Biol Chem $2000 ; 275$ : 22728-35.

4. Goldfinger LE, Stack MS, Jones JC. Processing of laminin-5 and its functional consequences: role of plasmin and tissuetype plasminogen activator. J Cell Biol 1998 ; $141: 255-65$.

5. Tsubota $Y$, Mizushima H, Hirosaki T, Higashi S, Yasumitsu H, Miyazaki K. Isolation and activity of proteolytic fragment of laminin-5 alpha3 chain. Biochem Biophys Res Commun 2000; 278 : 614-20.

6. Sasaki T, Gohring W, Mann $K$, et al. Short arm region of laminin-5 gamma 2 chain: structure, mechanism of processing and binding to heparin and proteins. J Mol Biol 2001 ; 314 : 751-63.

7. Gagnoux-Palacios $L$, Allegra M, Spirito F, et al. The short arm of the laminin gamma 2 chain plays a pivotal role in the incorporation of laminin 5 into the extracellular matrix and in cell adhesion. J Cell Biol 2001 ; $153: 835-50$.

8. Rousselle P, Keene DR, Ruggiero F, Champliaud MF, Rest M, Burgeson RE. Laminin 5 binds the NC-1 domain of type VII collagen. J Cell Biol 1997 ; 138: 719-28.

9. Kreidberg JA. Functions of alpha3betal integrin. Curr Opin Cell Biol 2000 ; 12 : 548-53.

10. Nievers MG, Schaapveld RP, Sonnenberg A. Biology and function of hemidesmosomes. Matrix Biol 1999 ; 18 : 5-17.

11. Ryan MC, Lee K, Miyashita Y, Carter WG. Targeted disruption of the LAMA3 gene in mice reveals abnormalities in survival and late stage differentiation of epithelial cells. J Cell Biol 1999 ; 145 : 1309-23.

12. Ghosh S, Stack MS. Proteolytic modification of laminins: functional consequences. Microsc Res Tech $2000 ; 51:$ 238-46.

13. Rousselle P, Lunstrum GP, Keene DR, Burgeson RE. Kalinin: an epitheliumspecific basement membrane adhesion molecule that is a component of anchoring filaments. J Cell Biol 1991; 114 : 567-76.

14. Ryan MC, Tizard R, VanDevanter DR, Carter WG. Cloning of the LamA3 gene encoding the alpha 3 chain of the adhesive ligand epiligrin. Expression in wound repair. J Biol Chem 1994 ; 269 : 22779-87.

15. Nguyen BP, Gil SG, Carter WG. Deposition of laminin 5 by keratinocytes regulates integrin adhesion and signaling. J Biol Chem 2000 ; 275 : 31896-907.

16. Décline F, Rousselle P. Keratinocyte migration requires alpha2betal integrin-mediated interactions with the laminin 5 gamma 2 chain. J Cell Sci 2001 ; 114 : 811-23.

17. Utani A, Nomizu M, Matsuura $\mathrm{H}$, et al. A unique sequence of the laminin alpha $3 \mathrm{G}$ domain binds to heparin and promotes cell adhesion through syndecan-2 and -4 . J Biol Chem 2001 ; 276 : 28779-88. 
18. Fukushima $Y$, Ohnishi T, Arita N, Hayakawa T, Sekiguchi, K. Integrin alpha3betal-mediated interaction with laminin-5 stimulates adhesion, migration and invasion of malignant glioma cells. Int J Cancer 1998 ; $76: 63-72$.

19. Koshikawa N, Giannelli G, Cirulli V, Miyazaki K, Quaranta V. Role of cell surface metalloprotease MT1-MMP in epithelial cell migration over laminin-5. J Cell Biol 2000 ; 148 : 615-24.

20. Giannelli G, Falk-Marzillier J, Schiraldi 0, StetlerStevenson WG, Quaranta V. Induction of cell migration by matrix metalloprotease2 cleavage of laminin- 5 . Science 1997 ; $277: 225-8$.

21. Miyazaki K, Kikkawa $Y$, Nakamura A, Yasumitsu $\mathrm{H}$,
Umeda M. A large celladhesive scatter factor secreted by human gastric carcinoma cells. Proc Natl Acad Sci. USA 1993 ; 90 : 11767-71.

22. Hirosaki T, Mizushima H, Tsubota Y, Moriyama K, Miyazaki K. Structural requirement of carboxylterminal globular domains of laminin alpha 3 chain for promotion of rapid cell adhesion and migration by laminin-5. J Biol Chem $2000 ; 275$ : 22495-502.

23. Shang M, Koshikawa N, Schenk S, Quaranta V. The LG3 module of laminin-5 harbors a binding site for integrin alpha3betal that promotes cell adhesion, spreading, and migration. J Biol Chem 2001; 276 : 33045-53.

24. Skyldberg B, Salo S,
Eriksson $\varepsilon$, et al. Laminin- 5 as a marker of invasiveness in cervical lesions. J Natl Cancer Inst 1999 ; 91 : 1882-7.

25. Pyke C, Romer J, Kallunki $P$, et al. The gamma 2 chain of kalinin/laminin 5 is preferentially expressed in invading malignant cells in human cancers. Am J Pathol 1994 ; 145 : 782-91.

26. Tani T, Lumme A, Linnala $A$, et al. Pancreatic carcinomas deposit laminin-5, preferably adhere to laminin-5, and migrate on the newly deposited basement membrane. Am J Pathol 1997 ; 151 : 1289-302.

27. Hao J, Yang Y, McDaniel KM, Dalkin BL, Cress AE, Nagle RB. Differential expression of laminin 5 (alpha 3-beta 3 -gamma 2) by human malignant and normal prostate. Am J Pathol 1996 ; 149 : 1341-9.

28. Sordat I, Bosman FT, Dorta $G$, et al. Differential expression of laminin-5 subunits and integrin receptors in human colorectal neoplasia. J Pathol 1998 ; 185 : 44-52.

29. Sordat I, Rousselle P, Chaubert $P$, et al. Tumor cell budding and laminin- 5 expression in colorectal carcinoma can be modulated by the tissue micro-environment. Int I Cancer $2000 ; 88$ : 708-17.

\section{TIRÉS À PART}

P. Rousselle 